\title{
Parasitic wasp responses to symbiont-based defense in aphids
}

\author{
Kerry M Oliver ${ }^{1 *}$, Koji Noge ${ }^{2}$, Emma M Huang ${ }^{3}$, Jaime M Campos ${ }^{3}$, Judith X Becerra ${ }^{4}$ and Martha S Hunter ${ }^{3}$
}

\begin{abstract}
Background: Recent findings indicate that several insect lineages receive protection against particular natural enemies through infection with heritable symbionts, but little is yet known about whether enemies are able to discriminate and respond to symbiont-based defense. The pea aphid, Acyrthosiphon pisum, receives protection against the parasitic wasp, Aphidius ervi, when infected with the bacterial symbiont Hamiltonella defensa and its associated bacteriophage APSE (Acyrthosiphon pisum secondary endosymbiont). Internally developing parasitoid wasps, such as A. ervi, use maternal and embryonic factors to create an environment suitable for developing wasps. If more than one parasitoid egg is deposited into a single aphid host (superparasitism), then additional complements of these factors may contribute to the successful development of the single parasitoid that emerges.

Results: We performed experiments to determine if superparasitism is a tactic allowing wasps to overcome symbiont-mediated defense. We found that the deposition of two eggs into symbiont-protected aphids significantly increased rates of successful parasitism relative to singly parasitized aphids. We then conducted behavioral assays to determine whether $A$. ervi selectively superparasitizes $H$. defensa-infected aphids. In choice tests, we found that A. ervi tends to deposit a single egg in uninfected aphids, but two or more eggs in $\mathrm{H}$. defensa-infected aphids, indicating that oviposition choices may be largely determined by infection status. Finally, we identified differences in the quantity of the trans- $\beta$-farnesene, the major component of aphid alarm pheromone, between $\mathrm{H}$. defensa-infected and uninfected aphids, which may form the basis for discrimination.

Conclusions: Here we show that the parasitic wasp A. ervi discriminates among symbiont-infected and uninfected aphids, and changes its oviposition behavior in a way that increases the likelihood of overcoming symbiont-based defense. More generally, our results indicate that natural enemies are not passive victims of defensive symbionts, and that an evolutionary arms race between A. pisum and the parasitoid A. ervi may be mediated by a bacterial symbiosis.
\end{abstract}

\section{Background}

Insects and other arthropods are frequently infected with heritable bacterial symbionts [1]. Theory predicts that these strictly inherited microbes must confer net benefits or manipulate host reproduction to invade and persist in host populations [2-4]. While documented fitness benefits of symbionts have been primarily nutritional, a number of recent studies have reported protective effects of symbiont infection, suggesting that defense against natural enemies may be another major route facilitating invasion of heritable symbionts into host populations [5-7]. Insect symbionts of diverse

\footnotetext{
* Correspondence: kmoliver@uga.edu

'Department of Entomology, University of Georgia, Athens, GA 30605, USA Full list of author information is available at the end of the article
}

bacterial lineages have been shown to provide substantial protection against fungal pathogens $[8,9]$, viruses $[10,11]$, predators [12,13], parasitoids [14-17] and parasitic nematodes [18]. Despite increasing awareness of the roles of these bacteria in protecting hosts, little is known about natural enemy responses to this line of defense. Just as herbivorous insects have evolved strategies to overcome plant chemical defenses, natural enemies may employ strategies to counter symbiont-based defense.

The pea aphid, Acyrthosiphon pisum, has become a model for characterizing the effects of infection with symbionts [19]. In addition to carrying the obligate nutritional symbiont, Buchnera aphidicola, this aphid is often infected with additional facultative bacteria, called
C Biomed Central

(C) 2012 Oliver et al; licensee BioMed Central Ltd. This is an Open Access article distributed under the terms of the Creative Commons Attribution License (http://creativecommons.org/licenses/by/2.0), which permits unrestricted use, distribution, and reproduction in any medium, provided the original work is properly cited. 
'secondary' symbionts, which have been found to mediate important ecological interactions. These effects include thermal tolerance [20,21], resistance to fungal pathogens [9] and parasitoid wasps [14]. In the latter case, $A$. pisum acquires partial to complete immunity to parasitism by the common parasitoid wasp Aphidius ervi via infection with the $\gamma$-proteobacterial symbiont Hamiltonella defensa [14,22]. In no-choice assays, wasps readily parasitized $H$. defensa-infected aphids, but failed to complete development in resistant aphids [14]. While the details of the protective mechanism of $H$. defensa remain unclear, a bacteriophage called APSE (Acyrthosiphon pisum secondary endosymbiont) is required to produce the protective phenotype, suggesting a key role for eukaryotic toxins carried by the phage $[23,24]$. Among examined lines, $A$. pisum lacking $H$. defensa (or those carrying $H$. defensa that lack APSE) are highly susceptible to parasitism by $A$. ervi, while lines carrying APSE-infected $H$. defensa receive partial to complete protection depending on $H$. defensa strain and associated phage haplotype [22-24].

Given the low probability of survival for wasp eggs laid in aphids infected with $H$. defensa and APSE, wasps should benefit from strategies that allow them to avoid or overcome symbiont-mediated defense. One potential strategy involves the use of dose-dependent factors associated with the number of oviposition events in a particular host [25]. Endoparasitoid wasps such as $A$. ervi typically employ both maternal and embryonic factors to counter host defenses and create an environment suitable for wasp development [26]. During oviposition, for example, female $A$. ervi wasps inject a $\gamma$-glutamyl transpeptidase-containing venom, which targets and degenerates pea aphid ovarioles, leading to a reduction in aphid fecundity and presumably enhancing resource availability for developing wasps $[27,28]$. Also, fast-growing polyploid cells called teratocytes dissociate from the serosal membrane surrounding the wasp embryo and circulate within the aphid hemocoel during larval development [29]. In $A$. ervi, teratocytes are known to synthesize and release two proteins that are likely involved in redirecting host resources from aphids to developing wasps: a fatty acid binding protein ( $A e-\mathrm{FABP})$, and an extracellular enolase $(A e-\mathrm{ENO})$, which may degrade aphid tissue $[30,31]$. If an $A$. ervi female oviposited twice in a single host, she would then double the quantity of both venom and teratocytes present in the host, potentially increasing the likelihood of successful parasitism in symbiontdefended hosts. Aphidius ervi is a solitary endoparasitoid; regardless of the numbers of eggs deposited, only one parasitoid will emerge from an aphid host. Nonetheless, in a host with more than one egg, the surviving wasp may gain an advantage in the host-parasitoid conflict from additional complements of venom and teratocytes.
In this study we examined potential wasp responses to symbiont-mediated defense in A. pisum. We conducted experiments to determine if superparasitism, defined here as the deposition of more than one egg, increases the likelihood of successful parasitism in symbiontdefended aphids. We also conducted a choice test to determine whether female $A$. ervi wasps' oviposition behavior differs when presented with both $H$. defensa infected and uninfected $A$. pisum. Lastly, we analyzed the volatile signature of $H$. defensa-infected and uninfected aphids as wasps could potentially exploit volatile differences occurring between symbiont infected and uninfected hosts to modify oviposition behavior when faced with symbiont-protected hosts. In bark beetles, for example, natural enemies use symbiont-derived volatiles to locate hosts [32,33].

\section{Results}

Is superparasitism a strategy to overcome symbiontbased defense?

We conducted parasitism assays of singly versus doubly parasitized aphids to determine if the deposition of two eggs (that is, superparasitism) versus one increased the likelihood of successful parasitism of aphids harboring defensive symbionts. We examined the effects of superparasitism on a range of experimental lines that varied in aphid genotype, infection status and $H$. defensa strain (Table 1). As expected from earlier studies [14,22], aphid lines infected with $H$. defensa received significant protection when singly parasitized by $A$. ervi relative to uninfected aphids sharing the same clonal background (Figure 1A, B, Table 2A. The amount of protection varied significantly (Table 2B) and depended upon $H$. defensa strain, with the $82 \mathrm{~B}$ strain, which carries APSE2 (encoding $c d t B$ toxin) (Table 1 ), conferring moderate protection in both aphid backgrounds (5A and A2E), and the two strains (A1A, A2F) carrying APSE-3 (encoding YD-repeat toxin) conferring high levels of protection (Figure 1). We also found that superparasitism (the deposition of two eggs) resulted in higher rates of successful parasitism compared to single parasitism (deposition of one egg) in all lines infected with $H$. defensa (Table 2C, Figure 1A, B). The increase in successful parasitism was observed in both aphid backgrounds and among all three $H$. defensa strains, which vary with respect to the phage haplotypes (APSE-2 and 3 ) carried. Between the two uninfected clones (5A and A2E) there was no overall effect of superparasitism on rates of successful parasitism in uninfected clones (Table 2C), but when considered individually the results appeared to vary. In uninfected clone $5 \mathrm{~A}$, double-parasitism did not lead to a significant increase in the proportion successfully parasitized, while it did in clone A2E. This difference between single and double 
Table 1 Experimental lines of A.pisum used in this study

\begin{tabular}{cccccc}
\hline A. pisum line & $\begin{array}{c}\text { H. defensa } \\
\text { strain }\end{array}$ & APSE- haplotype & $\begin{array}{c}\text { phage } \\
\text { toxin }\end{array}$ & $\begin{array}{c}\text { Clone } \\
\text { color }\end{array}$ & Collection Info: aphid clone (C) or symbiont donor (D) \\
\hline $5 \mathrm{~A}$ & none & & pink & C: Madison, WI 1999 \\
\hline $82 \mathrm{~B} \rightarrow 5 \mathrm{~A}$ & $82 \mathrm{~B}$ & APSE2 & $c d t B$ & pink & D: Cayuga Co., NY 2000 \\
\hline $\mathrm{A} 1 \mathrm{~A} \rightarrow 5 \mathrm{~A}$ & $\mathrm{~A} 1 \mathrm{~A}$ & APSE3 & YD-repeat & pink & D: Logan UT 2004 \\
\hline $\mathrm{A} 2 \mathrm{~F} \rightarrow 5 \mathrm{~A}$ & $\mathrm{~A} 2 \mathrm{~F}$ & APSE3 & YD-repeat & pink & D: Logan UT 2004 \\
\hline $\mathrm{A} 2 \mathrm{E}$ & none & & & green & C: Logan UT 2004 \\
\hline $82 \mathrm{~B} \rightarrow \mathrm{A} 2 \mathrm{E}$ & $82 \mathrm{~B}$ & APSE2 & $c d t B$ & green & D: Cayuga Co., NY 2000 \\
\hline
\end{tabular}

parasitism in clone A2E is unexpected if double-parasitism acts to only overcome $H$. defensa-associated resistance. However, upon closer examination, there was no increase in the number of mummies produced following double parasitism of A2E (t-test $P=0.60$ ), just fewer surviving aphids overall, which skewed the proportion of successfully parasitized aphids. In all $H$. defensa-infected strains, on the other hand, we found significant increases in the number of mummies when aphids were superparasitized. When doubly-parasitized, we found a $45 \%$ increase in mummies in line $82 \mathrm{~B} \rightarrow 5 \mathrm{~A}$, a $200 \%$ increase in mummies in $\mathrm{A} 1 \mathrm{~A} \rightarrow 5 \mathrm{~A}$, a $157 \%$ increase in $\mathrm{A} 2 \mathrm{~F} \rightarrow 5 \mathrm{~A}$, and a $32 \%$ increase in $82 \mathrm{~B} \rightarrow \mathrm{A} 2 \mathrm{E}$. Overall, we did not detect significant increases in aphid mortality in superparasitized versus singly parasitized cohorts across all treatments (ANOVA $\mathrm{F}_{1,119}=0.25 P=0.61$ ) or when restricted to $H$. defensa-infected lines (ANOVA $F_{1,79}=$ $2.21, P=0.14)$. Together this indicates that the significant increases in successful parasitism rates of superparasitized aphids in $H$. defensa-infected aphids was due primarily to greater production of mummies and not increased aphid mortality. Finally, in our logistic regression model, we found a significant interaction between infection status (infected vs. uninfected) and parasitism treatment (one vs. two oviposition events) among all lines (Likelihood effects ratio test, $\mathrm{df}=5, \mathrm{X}^{2}=11.8, P=$ 0.047 ) indicating that superparasitism led to a greater increase in successful parasitism in $H$. defensa-infected lines compared to uninfected lines.

\section{Number of eggs and teratocytes in singly versus doubly parasitized aphids}

We dissected parasitized aphids to verify that the number of observed parasitism events (single vs. double) corresponds to the number of eggs deposited in a single aphid host. In singly-parasitized $A$. pisum we found one egg in $86 \%(69 / 80$ ) aphids (two eggs in 0/80, no eggs in $11 / 80)$. In doubly-parasitized aphids we detected two eggs in $83 \%$ aphids (66/80) (one egg in 13/80, no eggs $1 / 80)$. In the aphids containing one fewer $A$. ervi egg than expected, it is unclear whether no egg was deposited or if we just did not find it. Regardless, these results

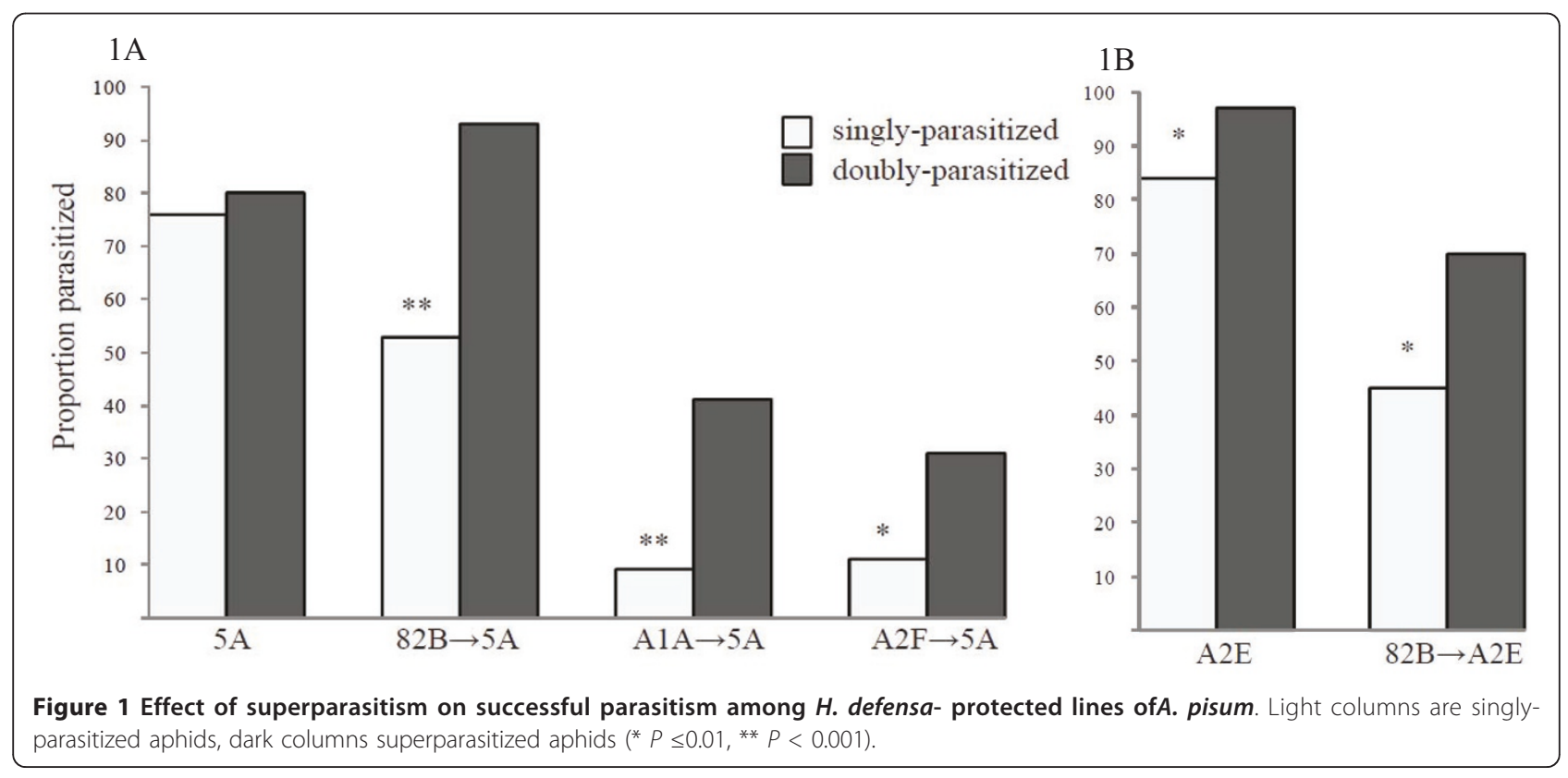


Table 2 Logistic regression analyses: regression equation is $Y=\beta_{0}+\beta_{1}+\beta_{2}$

\begin{tabular}{|c|c|c|}
\hline \multicolumn{3}{|c|}{$\begin{array}{l}\text { A. Resistance effects of infection with } H \text {. defensa relative to } \\
\text { uninfected control }\end{array}$} \\
\hline assay & Regression equation & $P$-value \\
\hline $5 \mathrm{~A}$ vs. $82 \mathrm{~B} \rightarrow 5 \mathrm{~A}$ & $Y=0.66-0.49^{\mathrm{Hd}}$ & $P=0.02$ \\
\hline $5 \mathrm{~A}$ vs. $\mathrm{A} 1 \mathrm{~A} \rightarrow 5 \mathrm{~A}$ & $Y=-0.57-1.73^{\mathrm{Hd}}$ & $P=0.0001$ \\
\hline $5 \mathrm{~A}$ vs. $\mathrm{A} 2 \mathrm{~F} \rightarrow 5 \mathrm{~A}$ & $Y=-0.57-1.72^{\mathrm{Hd}}$ & $P=0.0001$ \\
\hline A2E vs. $82 B \rightarrow A 2 E$ & $Y=0.69-0.90^{\mathrm{Hd}}$ & $P=0.001$ \\
\hline \multicolumn{3}{|c|}{$\begin{array}{l}\text { B. Variation in resistance among } H \text {. defensa strains in common } \\
\text { background } 5 \mathrm{~A}\end{array}$} \\
\hline A2F vs. $82 B$ vs. $A 1 A$ & $\mathrm{Y}=-1.13+1.3^{82 \mathrm{~B}}-0.16^{\mathrm{A} 1 \mathrm{~A}}$ & $P=0.001$ \\
\hline \multicolumn{3}{|c|}{ C. effects of superparasitism on rates of successful parasitism } \\
\hline Assay & Regression equation & $P$-value \\
\hline $5 \mathrm{~A}$ (uninfected) & $Y=1.28+0.13^{D P}$ & $P=0.57$ \\
\hline $82 \mathrm{~B} \rightarrow 5 \mathrm{~A}(\mathrm{H}$. defensa + APSE-2) & $Y=1.44+1.27^{D P}$ & $P=0.001$ \\
\hline $\mathrm{A} 1 \mathrm{~A} \rightarrow 5 \mathrm{~A}(\mathrm{H}$. defensa + APSE-3) & $Y=-1.34+0.97^{D P}$ & $P=0.0005$ \\
\hline $\mathrm{A} 2 \mathrm{~F} \rightarrow 5 \mathrm{~A}(\mathrm{H}$. defensa $+\mathrm{APSE}-3)$ & $Y=-1.55+0.73^{D P}$ & $P=0.004$ \\
\hline A2E (uninfected) & $Y=2.54+0.94^{D P}$ & $P=0.01$ \\
\hline $82 \mathrm{~B} \rightarrow \mathrm{A} 2 \mathrm{E}(\mathrm{H}$. defensa + APSE-2) & $Y=0.34+0.55^{D P}$ & $P=0.008$ \\
\hline All treatments & $Y=0.35+0.40^{D P}$ & $P<0.0001$ \\
\hline All H. defensa-infected & $Y=-0.26+0.46^{D P}$ & $P<0.0001$ \\
\hline All uninfected & $Y=1.71+0.32^{D P}$ & $P=0.07$ \\
\hline
\end{tabular}

$\mathrm{DP}$, double-parasitism; $\mathrm{Hd}, H$. defensa

indicate that one egg is typically deposited per parasitism event. We also examined whether double parasitism results in an extra complement of teratocytes. In uninfected line 5A, we found roughly twice the number of teratocytes in doubly parasitized aphids $(\mathrm{N}=8$, mean $45.8,95 \% \mathrm{CI}=41.7$ to 49.8$)$ relative to aphids parasitized once $(\mathrm{N}=8$, mean $23.1,95 \% \mathrm{CI}=19.1$ to 27.2$)$ indicating one complement of teratocytes is associated with each oviposition event. In singly parasitized, $H$. defensa-infected aphids (line $82 \mathrm{~B} \rightarrow 5 \mathrm{~A}$ ), however, we found a significant reduction (ANOVA $\mathrm{F}_{1,179}=71.2, P$ $<0.0001)$ in the number of teratocytes in aphids containing a dead or moribund larva $(\mathrm{N}=104$, mean $=$ $16.0,95 \% \mathrm{CI}=14.8$ to 17.2$)$ compared to aphids with a healthy larva $(\mathrm{N}=77$, mean $=23.7,95 \% \mathrm{CI} 22.4$ to 25.1) suggesting that a healthy complement of teratocytes may be important for wasp survival in a symbiontprotected host. We also observed that some teratocytes from this $H$. defensa-infected line $(82 \mathrm{~B} \rightarrow 5 \mathrm{~A})$ were irregular in shape, compared to those collected from uninfected aphids sharing the same genetic background (line $5 \mathrm{~A})$.

\section{Do wasps selectively superparasitize $H$. defensa-infected aphids?}

To determine if female $A$. ervi superparasitize $H$. defensa-infected aphids at a greater frequency than uninfected aphids, we conducted behavioral assays to monitor oviposition behavior of wasps. The pea aphid exhibits a pink-green color polymorphism, and we used clone color as a visual marker of infection status. We conducted two assays, each with an infected clone of one color/genotype paired with an uninfected clone of the other color/genotype. In choice tests, we found that female $A$. ervi wasps tended to lay a single egg in uninfected aphids while depositing more than one egg in $H$. defensa-infected aphids (Figure 2). The distribution of parasitism 'choices' (that is, no egg, one egg, > 1 egg) differed significantly between the green uninfected $(\mathrm{A} 2 \mathrm{E})$ and the pink $H$. defensa-infected $(\mathrm{A} 1 \mathrm{~A} \rightarrow 5 \mathrm{~A})$ (trial one, likelihood ratio test, $\mathrm{X}^{2}=43.5, P<0.0001$ ), as well as between the pink uninfected (5A) and green $H$. defensa-infected lines (trial two, likelihood ratio test, $\mathrm{X}^{2}$ $=30.2, P<0.0001)$. The majority of $H$. defensa-protected aphids, of both pink $(\mathrm{A} 1 \mathrm{~A} \rightarrow 5 \mathrm{~A})$ and green $(82 \mathrm{~B} \rightarrow \mathrm{A} 2 \mathrm{E})$ clones, were superparasitized, while the majority of uninfected $A$. pisum (5A = pink, $\mathrm{A} 2 \mathrm{E}=$ green) were singly parasitized. The distribution of parasitism choices did not vary between green (A2E) and pink (5A) uninfected clones (likelihood ratio test, $\mathrm{X}^{2}=$ 3.3, $P=0.2$ ), nor between green $H$. defensa-infected $(82 \mathrm{~B} \rightarrow \mathrm{A} 2 \mathrm{E})$ and pink $H$. defensa-infected $(\mathrm{A} 1 \mathrm{~A} \rightarrow 5 \mathrm{~A})$ lines (likelihood ratio test, $\mathrm{X}^{2}=1.8, P=0.4$ ). Together these results indicate that oviposition choices in these assays were largely determined by infection status rather than clone color.

\section{Compound identification and quantification using GC-MS} In whole aphid extracts, we identified significant differences in the quantity of trans- $\beta$-farnesene (EBF), between $H$. defensa-infected and uninfected aphids sharing the same genetic background (Figure 3). EBF, a volatile sesquiterpene, is the major component of the aphid alarm pheromone [34]. Aphids secrete alarm pheromone from cornicles when attacked. The pheromone alerts nearby aphids, which respond by walking away from the source of the pheromone, or by dropping from the food plant [35-37]. The alarm pheromone may also serve as a short-range host location cue used by both predators and parasitoids (reviewed in [38]). In uninfected aphid clone $5 \mathrm{~A}$, we detected $46.9(95 \% \mathrm{CI}=37.2$ to 56.5$) \mathrm{ng}$ of $\mathrm{EBF} / \mathrm{aphid}$ compared to $36.9 \mathrm{ng} \mathrm{EBF} / \mathrm{aphid}$ (95\% CI = 26.3 to 46.5$)$ in $H$. defensa-infected line $82 \mathrm{~B} \rightarrow 5 \mathrm{~A}$ and $29.5(95 \% \mathrm{CI}=19.8$ to 39.1$) \mathrm{ng} \mathrm{EBF} /$ aphid in $H$. defensa-carrying line $\mathrm{A} 1 \mathrm{~A} \rightarrow 5 \mathrm{~A}\left(\mathrm{ANOVA}_{2}, 30=3.46=\right.$ 0.04 ). All lines share the same aphid background so differences are due to infection status. We also used t-tests to evaluate each $H$. defensa-infected line relative to the uninfected control and found that EBF quantities in line $\mathrm{A} 1 \mathrm{~A} \rightarrow 5 \mathrm{~A}$ were significantly reduced relative to the control $(\mathrm{N}=11$, $\mathrm{t}$-test, $P=0.015)$, but that line $(82 \mathrm{~B} \rightarrow 5 \mathrm{~A})$ 


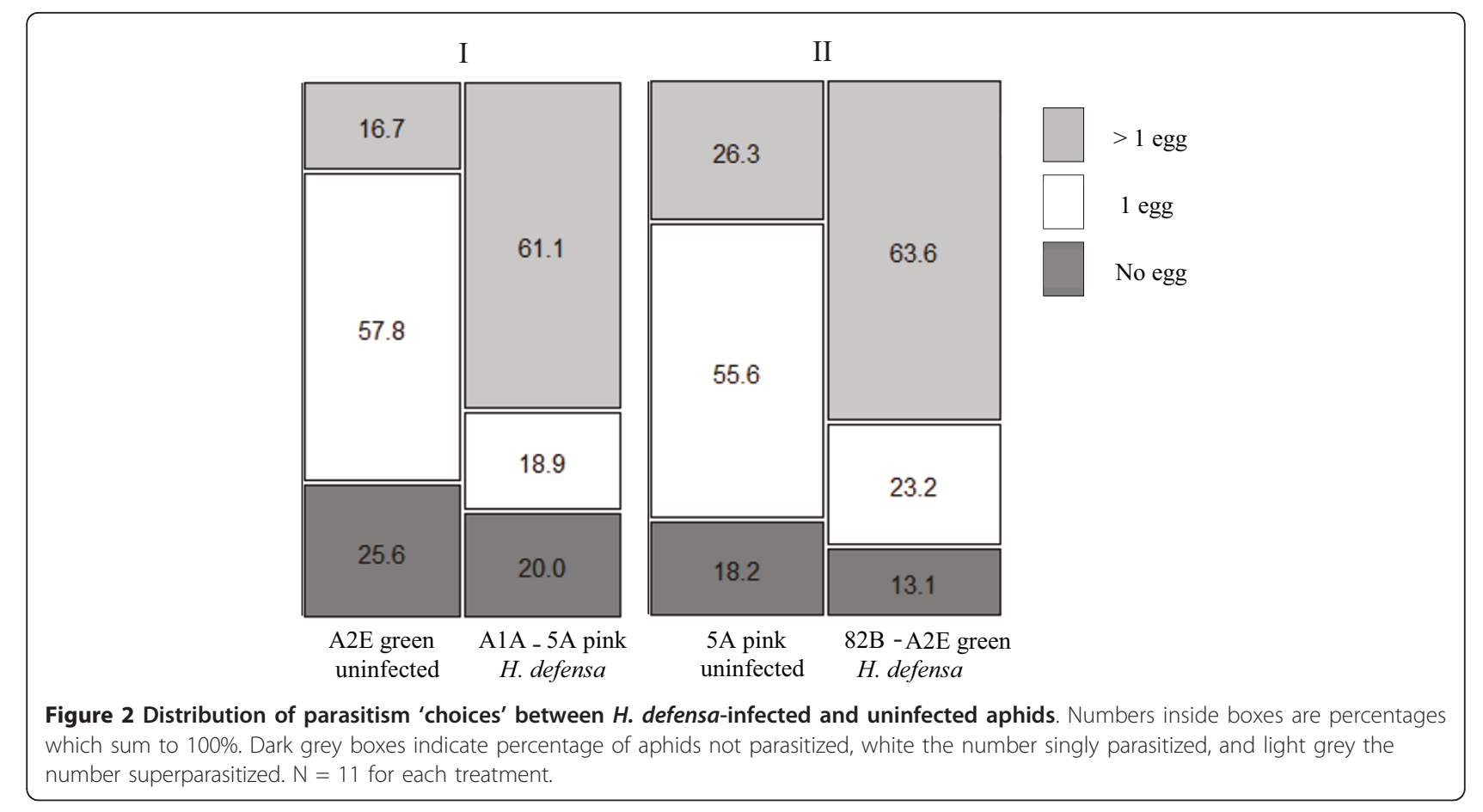

showed a non-significant reduction $(\mathrm{N}=11, P=0.18)$ relative to the control.

\section{Discussion}

Recent studies indicate that many insects are protected by heritable symbionts [7], yet little is known about how natural enemies respond to symbiont-based defenses. We report here that the parasitoid wasp, A. ervi, can partially overcome $H$. defensa-mediated protection in $A$. pisum by superparasitizing aphids (Figure 1, Table 2).

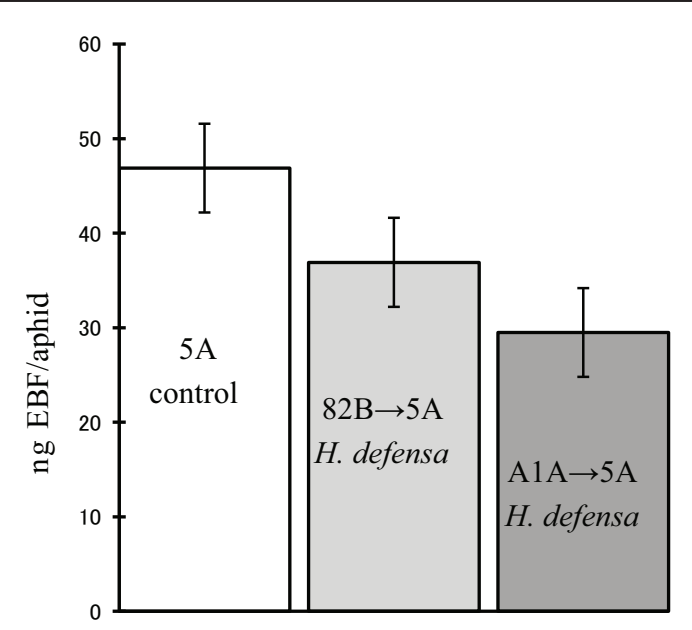

Figure 3 Amounts of trans- $\beta$-farnesene (EBF) per aphid in $\boldsymbol{H}$. defensa-infected versus uninfected $\boldsymbol{A}$. pisum. Bars $=\mathrm{SE}, \mathrm{N}=11$ each line.
Further, we find in choice assays that $A$. ervi can discriminate between $H$. defensa-infected and uninfected $A$. pisum and selectively superparasitizes infected aphids, while laying single eggs in uninfected hosts (Figure 2). Taken together, these results indicate that $A$. ervi has likely evolved successful strategies to partially counter symbiont-based defenses employed by $A$. pisum. Counter-strategies to overcome defense on the trophic level below are well known. Herbivorous insects have devised strategies for countering plant-based defenses (for example, [39-41]), and predators and parasitoids may overcome herbivore behavioral $[42,43]$ and chemical defenses as well. This report provides evidence that natural enemies are sometimes able to counteract symbiont-based defense. Recent reports have documented: 1 ) the evolution of increased $A$. ervi virulence when faced with symbiont-carrying resistant $A$. pisum lines [44] and 2) genotypic variation in the parasitoid Lysiphlebus fabarum's ability to successfully parasitize $H$. defensa-infected Aphis fabae (black bean aphid) [16]. The experimental design of these studies, however, does not allow conclusive partitioning of increased virulence towards symbiont- versus host-based defensive factors, but these reports do indicate that parasitic wasps likely have additional mechanisms for overcoming symbiontbased resistance.

Consistent with previous reports, we found that aphids infected with $H$. defensa, when singly parasitized, received moderate to high levels of protection from attack by $A$. ervi, depending upon strain [22]. We also 
found that the same strain of $H$. defensa (82B) in two backgrounds (A2E and 5A) conferred similar levels of protection, indicating little symbiont $\mathrm{X}$ aphid interaction [22]. When doubly-parasitized, however, we found that the likelihood of successful parasitism of $H$. defensainfected aphids increased in every instance, in both pink $(5 \mathrm{~A})$ and green (A2E) aphid backgrounds and among all three $H$. defensa strains (82B, A1A and A2F). Strains $\mathrm{A} 1 \mathrm{~A}$ and $\mathrm{A} 2 \mathrm{~F}$ carry the YD-repeat toxin-encoding phage variant ASPE-3 and strain 82B carries the $c d t B$ encoding APSE-2 [23]. Not only does superparasitism increase successful parasitism in symbiont defended hosts, but wasps gain a relatively greater benefit of superparasitism when attacking $H$. defensa infected aphids compared to uninfected aphids. Thus, superparasitism appears to be an effective counter strategy to both phage variants hitherto detected in N. American populations in A. pisum. One caveat regards the possibility that wasps emerging from successfully superparasitized aphids are not fit enough to reproduce themselves. While we did not explicitly examine wasp fitness, we did verify that adult wasps emerge from superparasitized hosts and bear no obvious abnormalities. A previous study in this system found slight increases in dry weight, and no differences in developmental time, in wasps emerging from superparasitized aphids relative to singly parasitized A. pisum [45], indicating that superparasitism does not generally lead to reductions in wasp fitness. In future experiments we will examine costs associated with superparasitism as a strategy to overcome defensive symbionts.

The mechanisms underlying the increase in successful parasitism of superparasitized aphids are not known, but the additional maternal and embryonic factors found in superparasitized hosts may contribute. In aphids infected with $82 \mathrm{~B} \rightarrow 5 \mathrm{~A}$, dead or moribund larvae appear four to five days post parasitism - long after teratocytes have dissociated from the extra-embryonic membrane [46]. We confirmed that an additional complement of teratocytes was present in doubly-parasitized aphids. We also found reductions in the number and irregularities in the morphology of teratocytes deposited in $H$. defensa infected aphids suggesting a possible interaction. Polyploid teratocytes are believed to be involved in redirecting resources from aphid to wasp development $[28,29]$. Fewer, or defective, teratocytes may prevent the creation of an environment suitable for wasp development. The extra complement of teratocytes in superparasitized aphids may shift the balance in favor of successful parasitoid development.

Some authors have suggested that the likelihood of one solitary parasitoid surviving host encapsulation responses may increase when multiple eggs are deposited in a single host -the so-called 'multiple target hypothesis' (for example, [47,48], rev. in [25]). While there are some accounts that host encapsulation responses are not as effective when faced with multiple parasitism events (for example, [49-51]), Vinson [52] reviewed the evidence for the multiple target hypothesis and concluded that target proliferation is probably not generally a viable strategy for endoparasitoids in overcoming host encapsulation defenses. While the aphid encapsulation response is very weak in our system, the notion of multiple parasitoid targets, especially teratocytes, diluting the effectiveness of symbiont-based defenses, may resurrect this hypothesis for cases when protective symbionts are present.

We found that not only does superparasitism increase the odds of successful parasitism, but that $A$. ervi females appear to discriminate between $H$. defensainfected aphids and uninfected aphids (Figure 2) and preferentially superparasitize symbiont-defended aphids. This indicates that wasps likely employ superparasitism as a strategy to overcome symbiont-based defense. In choice tests, females were more likely to lay single eggs in uninfected aphids, but more than one egg in $H$. defensa infected aphids. Since only one wasp can complete development in a single aphid, self-superparasitism (more than one egg laid by the same mother) by solitary endoparasitoids like $A$. ervi has generally been considered maladaptive, yet wide-ranging conditions have been proposed under which this strategy may be adaptive [51]. If the net fitness of offspring is higher under conditions of superparasitism compared to single-parasitism then selection may favor the sacrifice of an additional egg for a parasitoid such as $A$. ervi, which is limited by their time to find hosts rather than the number of eggs they have [25,53]. Aphidius ervi has a large number (96 to 567) of small, yolk-free eggs during adulthood (for example, [54,55]). While we did not compare the net fitness of wasps when singly parasitizing versus superparasitizing hosts, we did find large increases in the numbers of mummies (32 to 200\%) produced by superparasitism in each of the $H$. defensa-infected lines. This sharp increase in successful parasitism combined with the relatively low expected cost of $A$. ervi eggs suggests that superparasitism may produce net benefits. Thus, superparasitism may be an adaptive strategy when faced with symbiont-defended hosts.

We found that levels of trans- $\beta$-farnesene (EBF) were lower in $H$. defensa-infected aphids (Figure 3), and differences in the abundance of this volatile compound are a possible basis for wasp discrimination between infected and uninfected hosts. EBF is the major component of aphid alarm pheromone and is secreted from the cornicles (siphunculi). EBF in aphids has been found to serve as a short-range (due to high reactivity with ozone) attractant to a wide range of natural enemies, 
including both predators and parasitoids (rev. [38]). Cornicle secretions, although not specifically EBF, from $A$. pisum have been reported to be a contact kairomone for A. ervi [56]. Aphids release alarm pheromone when confronted with natural enemies, which often leads to escape behaviors, such as dropping from the host plant $[36,37]$, and inducing the production of winged morphs [57]. In groups of symbiont-defended aphids, the release of lower amounts of EBF could be adaptive, as dropping from the plant, or dispersing is risky [58] while staying put may be a better strategy if one is likely to survive a parasitoid attack. A recent report found that $A$. pisum infected with $H$. defensa exhibited fewer escape behaviors and reduced aggressiveness relative to uninfected counterparts [59], consistent with reduced levels of EBF. More generally, heritable variation in escape behavior among $A$. pisum clones has been reported [60]; and heritable symbiont influences on EBF titers could explain some of this variation.

In addition to documenting parasitoid behavioral responses to symbiont-based defense in herbivores, it is also remarkable that aphid secondary symbionts appear to be detectable by foraging parasitoids. Oviposition by Aphidius ervi, and aphidiine parasitoids in general, is extraordinary in its speed; the parasitoid faces the aphid, coils its abdomen underneath its head and thorax so that it too faces forward, and appears to strike the aphid with the ovipositor at the tip of its abdomen, generally laying an egg in that instant. Aphidiine wasps are not generally known to lay two eggs with a single strike $[45,61]$, so superparasitism involves a second strike and oviposition. Detection of contact or even internal compounds likely involve sensilla on the ovipositor [62] presumably after contact chemicals have been detected and evaluated. That detection of the host's condition can occur during this very short interval has been shown by several authors $[61,63,64]$; female $A$. ervi were shown to avoid parasitism of aphids already containing a parasitoid egg. We show that the levels of EBF are generally lower in symbiont-defended relative to uninfected $A$. pisum. Whether EBF is the compound used to discriminate between symbiont-defended and uninfected aphids, however, is not certain. Infection with $H$. defensa and other symbionts has been shown to alter the pool of metabolites in A. pisum [65], and these changes may also serve as host selection cues. It would be interesting, however, if symbiont-defended aphids use reduced EBF to increase the threshold for dropping behavior, but parasitoids have co-opted the signal for their own benefit.

\section{Conclusions}

Recent reports indicate that a wide range of insects are protected from natural enemies via infection with heritable symbionts, yet little is known about natural enemy responses to symbiont-based protection [7]. Here we report that the parasitic wasp $A$. ervi discriminates between symbiont-defended and undefended (that is, uninfected) aphids, and modifies its oviposition behavior when faced with protective symbionts in ways that increase the likelihood of successful parasitism. Wasps selectively deposit two or more eggs in symbiontdefended hosts, even though only one wasp can complete development within a single aphid. The discovery of wasp behavioral responses to symbiont-based defense in aphids sets the stage for further investigations of the coevolutionary dynamics of the host $A$. pisum and its parasitoid $A$. ervi, as mediated by a bacterial symbiosis. In general, we expect that the study of natural enemies that are confronted with defensive symbionts will yield insights into various counter-strategies to circumvent or overcome the protection these symbionts provide.

\section{Methods}

Aphids, heritable bacteria and experimental lines

Acyrthosiphon pisum (Hemiptera: Aphididae) is a phloem-feeding polyphagous pest of herbaceous legumes [66] that was introduced to North America from Europe around 1870. The pea aphid reproduces asexually, except in the autumn, when a shorter photoperiod induces the production of sexual morphs, which mate and produce diapausing eggs. By mimicking long-day length conditions (16:8 L:D) in the laboratory, clonal lineages can be maintained indefinitely. This aphid also exhibits a pink-green color polymorphism, with pink morphs encoding a carotenoid desaturase not present in green morphs [67]. Each $A$. pisum clonal line used in these experiments was comprised of descendants of a single parthenogenetic female maintained on Vicia faba (fava bean) at $20^{\circ} \mathrm{C}+/-1^{\circ} \mathrm{C}$, and 16:8 L:D in a biological incubator.

In this study, we used experimental lines created by microinjection for a previous study [22], which comprise combinations of three $H$. defensa strains in two $A$. pisum clonal backgrounds (Table 1 ). We use the term uninfected to refer to aphids (or clonal lines) that are uninfected with any secondary symbionts, but still retain Buchnera. We routinely screened lines with diagnostic PCR to ensure expected infection status as in Oliver et al. [22]. In our laboratory colonies the vertical transmission rate of $H$. defensa approaches $100 \%$, and infections established by microinjection have proven stable in cultures for up to 10 years. We also performed a diagnostic fingerprinting technique (intersequence simple repeats or ISSR) to verify aphid genotype [68].

\section{Parasitoids}

The common, solitary endoparasitoid $A$. ervi (Haliday) (Hymenoptera: Braconidae) was released in North America to control A. pisum populations. The adult 
female deposits an egg inside a suitable aphid nymph. After approximately $24 \mathrm{hrs}\left(\right.$ at $20^{\circ} \mathrm{C}$ ) the egg hatches and the resulting wasp larva develops within a living aphid for about one week. The parasitoid eventually kills the aphid, consumes the host viscera, and causes the aphid cuticle to transform into the characteristic 'mummy', in which the wasp pupates. A free-living adult $A$. ervi wasp emerges from the mummy. In the current study, the wasp culture was maintained in the laboratory at $20^{\circ} \mathrm{C}+/-1^{\circ} \mathrm{C}$ and $16 \mathrm{~L}: 8 \mathrm{D}$, on an $A$. pisum clone (5A) uninfected with $H$. defensa.

\section{Number of eggs and teratocytes in singly versus superparasitized aphids}

We dissected aphids approximately six hours (held at $20^{\circ} \mathrm{C}$ ) after parasitism in $1 \mathrm{X}$ PBS (phosphate-buffered saline) solution and searched for $A$. ervi eggs with the aid of a Leica MZ6 stereomicroscope (Wetzlar, Germany) to verify that single and double parasitism events $(\mathrm{N}=80$, each) result in one and two eggs, respectively, deposited per aphid host. To determine if superparasitism results in a double complement of teratocytes, we dissected singly- and doubly-parasitized third-instar aphids $(\mathrm{N}=8$ each, uninfected line $5 \mathrm{~A})$ approximately 96 hours $\left(20^{\circ} \mathrm{C}\right)$ after parasitism in $1 \mathrm{X}$ PBS to estimate teratocyte counts per host. We also investigated potential interactions between $H$. defensa and teratocytes by examining the number of teratocytes approximately 96 hours $\left(20^{\circ} \mathrm{C}\right)$ after parasitism in singly parasitized aphids containing either healthy (resistant $\mathrm{N}=77$ ) or dead/ moribund (susceptible, $\mathrm{N}=104$ ) wasp larvae in aphid line $82 \mathrm{~B} \rightarrow 5 \mathrm{~A}$.

Does superparasitism overcome symbiont-based defense? Each replicate of protection assays consisted of cohorts of 10 second-instar $A$. pisum being either singly- or doubly- parasitized by $A$. ervi in a Petri dish, placed on a potted $V$. faba plant in a cup cage, and then held in a biological incubator at $20^{\circ} \mathrm{C}+/-1^{\circ} \mathrm{C}$ and 16L:8D. We conducted 10 replicates of each treatment. Eclosion rates (adult wasps emerging from mummies) approach $100 \%$ for both singly and doubly-parasitized aphids. Since mummies are sessile (that is, easy to count accurately), and a suitable proxy to estimate parasitism rates, we counted the numbers of mummies and surviving aphids after 10 days to determine rates of successful parasitism. We conducted two assays. The first assay examined the effect of superparasitism on experimental lines $5 \mathrm{~A}, 82 \mathrm{~B} \rightarrow 5 \mathrm{~A}, \mathrm{~A} 1 \mathrm{~A} \rightarrow 5 \mathrm{~A}$, allowing us to examine effects on two different $H$. defensa strains with different phage haplotypes and associated toxins (Table 1). In assay 2, we examined the effects of superparasitism on experimental lines $\mathrm{A} 2 \mathrm{E}$ and $82 \mathrm{~B} \rightarrow \mathrm{A} 2 \mathrm{E}$, allowing us to examine effects in a distinct $A$. pisum background.
Parasitism assays were conducted at least 40 generations after artificial infection. Koga et al. [69] reported that detrimental effects of novel infections with S. symbiotica on $A$. pisum had attenuated by eight months post-infection. We analyzed the proportion of successfully parasitized aphids in a logistic regression (that is, computing $\log$ odds of mummification) framework testing for effects of infection status and parasitism treatment as well as calculating the interaction term between infection status and parasitism treatment. All statistical analyses were performed using JMP 9.02 software (SAS, Cary, North Carolina, USA).

\section{Do wasps selectively superparasitize $H$. defensa-infected aphids?}

To determine if female $A$, ervi superparasitize $H$. defensa-infected aphids at a greater frequency than uninfected aphids, we conducted behavioral assays to monitor oviposition behavior of wasps. In each section of a three-way divided Petri dish we placed a second, third and fourth instar nymph of each color morph (for example, uninfected green clone $=\mathrm{A} 2 \mathrm{E}$, and $H$. defensainfected pink clone $\mathrm{A} 1 \mathrm{~A} \rightarrow 5 \mathrm{~A}$ ) for a total of 18 aphids in each dish. The use of a subdivided dish, three instars, and two color morphs allowed us to identify every individual aphid and observe the number of times each aphid was parasitized. We also reversed the color scheme for half of the replicates, using the uninfected pink clone $(5 \mathrm{~A})$ and $H$. defensa-infected green clone $(82 \mathrm{~B} \rightarrow \mathrm{A} 2 \mathrm{E})$ to account for the possibility that parasitoids preferentially prefer to attack one color morph. For each replicate of the assay, we introduced a single, mated $A$. ervi female into the dish and we recorded the number of times that each individual aphid was parasitized. We allowed wasps to forage for 30 minutes or to lay a maximum of 24 eggs (whichever came first), to increase the likelihood that most aphids were examined as potential hosts. Wasps that attacked no aphids in the first five minutes after introduction to the arena were removed and replaced with another female. The experiment was conducted 'blind' as the observer was unaware which clone (green or pink) in each trial was $H$. defensa-infected. We exposed 378 aphids to wasps and recorded more than 500 attacks in 10 to 11 trials of each combination. We analyzed the proportion of aphids that were a) not parasitized, b) singly parasitized, or c) parasitized two or more times using a likelihood ratio test contingency analysis with clone color (pink or green), infection status ( $H$. defensa-infected or not), and the individual wasps as explanatory variables. Variation among individual wasps, which were never re-used, did not contribute significantly to the model, and this variable was removed from the model for subsequent analyses. 
Gas chromatography (GC) and mass spectrometry (MS) to identify volatile differences between $H$. defensa-infected and uninfected aphids

To determine whether there were detectable differences in the volatile signature of $H$. defensa-infected and uninfected aphids, replicate samples of five aphids of each type were collected in a glass tube, and were extracted with $100 \mu \mathrm{l}$ of dichloromethane with $5 \mathrm{ng} / \mu \mathrm{l}$ of 1 -dodecene as an internal standard (IS), for five minutes. We analyzed a two microliter sample using GC-MS carried out by an Agilent $6890 \mathrm{~N}$ gas chromatograph linked to an Agilent 5975B mass spectrometer operated at $70 \mathrm{eV}$ using a HP-5MS capillary column (Agilent Technologies (Santa Clara, California, USA), $30 \mathrm{~m} \times 0.25 \mathrm{~mm}$ ID, $0.25 \mu \mathrm{m}$ in film thickness) with helium carrier gas at a velocity of $1.2 \mathrm{ml} /$ minute in splitless mode. The oven temperature was held at $60^{\circ} \mathrm{C}$ for two minutes and programmed to increase at $10^{\circ} \mathrm{C} /$ minute from $60^{\circ} \mathrm{C}$ to $300^{\circ}$ $\mathrm{C}$ and then held at $300^{\circ} \mathrm{C}$ for five minutes. The injector temperature was maintained at $200^{\circ} \mathrm{C}$ and the detector temperature at $300^{\circ} \mathrm{C}$. One compound, trans- $\beta$-farnesene $(E B F)$, varied significantly among treatments. We verified the identity of this compound by comparing its $\mathrm{GC}$ retention time and mass spectrum with those of an authentic standard (Sigma-Aldrich, St Louis, Missouri, USA)). To quantify the amount of EBF in each sample, we performed GC analysis with a flame ionization detector (FID), using a J \& W DB-5MS capillary column (Agilent Technologies, Santa Clara, California, USA), 25 $\mathrm{m} \times 0.32 \mathrm{~mm}$ ID, $0.52 \mu \mathrm{m}$ in film thickness) under the same conditions as those for GC-MS, except that the velocity of helium carrier was $2.0 \mathrm{ml} /$ minute. To prepare the calibration curve for EBF, six known concentrations of the authentic standard were used $(0.5$ to $5 \mathrm{ng} / \mu \mathrm{l})$. The peak area of the compound was divided by that of the IS, and then a calibration curve for the compound was prepared using the peak area ratio and the known concentrations. The concentrations of the compound in samples were determined by comparing their peak area ratio with the one found in the calibration curve. The amounts of EBF recorded were normally distributed and we conducted an ANOVA and t-tests to compare means among/between treatments as well as to determine $(1-\alpha)$ 95\% confidence intervals using JMP 9.02 software (SAS, Cary, North Carolina, USA.

\section{Abbreviations \\ APSE: Acyrthosiphon pisum secondary endosymbiont; EBF: trans- $\beta$-farnesene; FID: flame ionization detector; ISSR: intersequence simple repeats; PBS: phosphate-buffered saline}

\section{Acknowledgements and funding}

We thank Nancy Moran for helpful discussions and comments on the manuscript. This project was supported by the National Research Initiative of the U.S. Department of Agriculture Cooperative Research, Education, and
Extension Service, grant 2006-35302-17307 to KMO and MSH and National Science Foundation (IOS) grant 1050128 to KMO.

\section{Author details}

${ }^{1}$ Department of Entomology, University of Georgia, Athens, GA 30605, USA. ${ }^{2}$ Department of Biological Production, Akita Prefectural University, Akita, Japan 010-0195. 를 AZ 85721, USA. ${ }^{4}$ Department of Biosphere 2, University of Arizona, Tucson, AZ 85721, USA.

\section{Authors' contributions}

$\mathrm{KMO}, \mathrm{JXB}$ and MSH designed the study. $\mathrm{KMO}, \mathrm{KN}, \mathrm{EMH}$ and JMC performed the research. KMO and MSH analyzed the data and wrote the paper. All authors read and approved the final manuscript.

\section{Competing interests}

The authors declare that they have no competing interests.

Received: 5 October 2011 Accepted: 24 February 2012

Published: 24 February 2012

\section{References}

1. Moran NA, McCutcheon JP, Nakabachi A: Genomics and evolution of heritable bacterial symbionts. Ann Rev Genet 2008, 42:165-190.

2. Bull JJ: Evolution of Sex Determining Mechanisms Menlo Park, California: Benjamin/Cummings; 1983.

3. Engelstadter J, Hurst GDD: The ecology and evolution of microbes that manipulate host reproduction. Annu Rev Ecol Evol Syst 2009, 40:127-149.

4. Werren JH, O'Neill SL: The evolution of heritable symbionts. In Influential passengers: inherited microorganisms and arthropod reproduction. Edited by: O'Neill SL, Hoffmann AA, Werren JH. Oxford, UK: Oxford University Press; 1997:1-41.

5. Brownlie JC, Johnson KN: Symbiont-mediated protection in insect hosts. Trends Microbiol 2009, 17:348-354.

6. Haine ER: Symbiont-mediated protection. ProcR Soc B-Biol Sci 2008 , 275:353-361

7. Oliver KM, Moran NA: Defensive symbionts in aphids and other insects. In Defensive Mutualism in Microbial Symbiosis. Edited by: White JF, Torres MS. London: Taylor 2009:129-148.

8. Kaltenpoth M, Gottler W, Herzner G, Strohm E: Symbiotic bacteria protect wasp larvae from fungal infestation. Curr Biol 2005, 15:475-479.

9. Scarborough $\mathrm{CL}$, Ferrari J, Godfray $\mathrm{HCJ}$ : Aphid protected from pathogen by endosymbiont. Science 2005, 310:1781-1781.

10. Hedges LM, Brownlie JC, O'Neill SL, Johnson KN: Wolbachia and virus protection in insects. Science 2008, 322:702-702.

11. Teixeira L, Ferreira A, Ashburner M: The bacterial symbiont Wolbachia induces resistance to RNA viral infections in Drosophila melanogaster. Plos Biology 2008, 6:2753-2763.

12. Kellner RLL, Dettner K: Differential efficacy of toxic pederin in deterring potential arthropod predators of Paederus (Coleoptera: Staphylinidae) offspring. Oecologia 1996, 107:293-300.

13. Piel J: A polyketide synthase-peptide synthetase gene cluster from an uncultured bacterial symbiont of Paederus beetles. Proc Natl Acad Sci U Sf A 2002, 99:14002-14007.

14. Oliver KM, Russell JA, Moran NA, Hunter MS: Facultative bacterial symbionts in aphids confer resistance to parasitic wasps. Proc Natl Acad Sci USA 2003, 100:1803-1807.

15. Vorburger C, Gehrer L, Rodriguez P: A strain of the bacterial symbiont Regiella insecticola protects aphids against parasitoids. Biol Lett 2010, 6:109-111.

16. Vorburger C, Sandrock C, Gouskov A, Castaneda LE, Ferrari J: Genotypic variation and the role of defensive endosymbionts in an all parthogenetic host-parasitoid interaction. Evolution 2009, 63:1439-1450.

17. Xie JL, Vilchez I, Mateos M: Spiroplasma bacteria enhance survival of Drosophila hydei attacked by the parasitic wasp Leptopilina heterotoma. Plos One 2010, 5:e12149.

18. Jaenike J, Unckless R, Cockburn SN, Boelio LM, Perlman SJ: Adaptation via symbiosis: recent spread of a Drosophila defensive symbiont. Science 2010, 329:212-215.

19. Oliver KM, Degnan PH, Burke GR, Moran NA: Facultative symbionts in aphids and the horizontal transfer of ecologically important traits. Ann Rev Entomol 2010, 55:247-266. 
20. Montllor CB, Maxmen A, Purcell AH: Facultative bacterial endosymbionts benefit pea aphids Acyrthosiphon pisum under heat stress. Ecol Entomol 2002, 27:189-195.

21. Russell JA, Moran NA: Costs and benefits of symbiont infection in aphids: variation among symbionts and across temperatures. Proc R Soc B-Biol Sci 2006, 273:603-610

22. Oliver KM, Moran NA, Hunter MS: Variation in resistance to parasitism in aphids is due to symbionts not host genotype. Proc Natl Acad Sci USA 2005, 102:12795-12800

23. Degnan $\mathrm{PH}$, Moran NA: Diverse phage-encoded toxins in a protective insect endosymbiont. Appl Environ Microbiol 2008, 74:6782-6791.

24. Oliver KM, Degnan PH, Hunter MS, Moran NA: Bacteriophages encode factors required for protection in a symbiotic mutualism. Science 2009, 325:992-994.

25. Godfray HCJ: Parasitoids: Behavioral and Evolutionary Ecology Princeton: Princeton University Press; 1994.

26. Beckage NE, Gelman DB: Wasp parasitoid disruption of host development: Implications for new biologically based strategies for insect control. Ann Rev Entomol 2004, 49:299-330.

27. Digilio MC, Isidoro N, Tremblay E, Pennacchio F: Host castration by Aphidius ervi venom proteins. J Insect Physiol 2000, 46:1041-1050.

28. Falabella P, Riviello L, Caccialupi P, Rossodivita T, Valente MT, De Stradis ML, Tranfaglia A, Varricchio P, Gigliotti S, Graziani F, Malva C, Pennacchio F: A gamma-glutamyl transpeptidase of Aphidius ervi venom induces apoptosis in the ovaries of host aphids. Insect Biochem Mol Biol 2007, 37:453-465.

29. Falabella P, Tremblay E, Pennacchio F: Host regulation by the aphid parasitoid Aphidius ervi: the role of teratocytes. Entomol Exp Appl 2000, 97:1-9.

30. Falabella P, Perugino G, Caccialupi P, Riviello L, Varricchio P, Tranfaglia A, Rossi M, Malva C, Graziani F, Moracci M, Pennacchio F: A novel fatty acid binding protein produced by teratocytes of the aphid parasitoid Aphidius ervi. Insect Mol Biol 2005, 14:195-205.

31. Falabella P, Riviello L, De Stradis ML, Stigliano C, Varricchio P, Grimaldi A, de Eguileor M, Graziani F, Gigliotti S, Pennacchio F: Aphidius ervi teratocytes release an extracellular enolase. Insect Biochem Mol Biol 2009, 39:801-813.

32. Boone CK, Six DL, Zheng YB, Raffa KF: Parasitoids and dipteran predators exploit volatiles from microbial symbionts to locate bark beetles. Environ Entomol 2008, 37:150-161.

33. Sullivan BT, Berisford CW: Semiochemicals from fungal associates of bark beetles may mediate host location behavior of parasitoids. J Chem Ecol 2004, 30:703-717.

34. Bowers WS, Webb RE, Nault LR, Dutky SR: Aphid alarm pheromone -isolation, identification, synthesis. Science 1972, 177:1121-1122.

35. Kislow CJ, Edwards LJ: Repellent odor in aphids. Nature 1972, 235:108-109.

36. Montgomery ME, Nault LR: Aphid alarm pheromones - dispersion of Hyadaphis erysimi and Myzus persicae (Hemiptera-Aphididae). Ann Entomol Soc Am 1977, 70:669-672.

37. Nault $L R$, Edwards LJ, Styer WE: Aphid alarm pheromones: secretion and reception. Environ Entomol 1973, 2:101-105.

38. Hatano E, Kunert G, Michaud JP, Weisser WW: Chemical cues mediating aphid location by natural enemies. Eur J Entomol 2008, 105:797-806.

39. Becerra JX: Squirt-gun defense in Bursera and the chrysomelid counterploy. Ecology 1994, 75:1991-1996.

40. Brattsten LB, Wilkinson CF, Eisner T: Herbivore-plant interactions: mixedfunction oxidases and secondary plant substances. Science 1977, 196:1349-1352.

41. Glendinning ال ال How do herbivorous insects cope with noxious secondary plant compounds in their diet? Entomol Exp pp/ 2002, 104:15-25.

42. Yeargan KV, Braman SK: Life-history of the parasite Diolcogaster facetosal (Weed) (Hymenoptera, Braconidae) and its behavioral adaptation to the defenseive response of a lepidopteran host. Ann Entomol Soc Am 1986, 79:1029-1033.

43. Yeargan KV, Braman SK: Life-history of the hyperparasitoid Mesochorus discitergus (Hymenoptera, Ichneumondiae) and tactics used to overcome the defensive behavior of the green cloverworm (Lepidoptera, Noctuidae). Ann Entomol Soc Am 1989, 82:393-398.

44. Dion E, Zele F, Simon JC, Outreman Y: Rapid evolution of parasitoids when faced with the symbiont-mediated resistance of their hosts. J Evol Biol 2011, 24:741-750.
45. Bai B, Mackauer M: Influence of superparasitism on development rate and adult size in a solitary parasitoid wasp, Aphdius ervi. Funct Ecol 1992, 6:302-307

46. Oliver KM, Moran NA, Hunter MS: Costs and benefits of a superinfection of facultative symbionts in aphids. Proc R Soc B-Biol Sci 2006, 273:1273-1280.

47. Askew RR: A survey of leaf-miners and their parasites on Laburnum. Trans R Entomol So Lond 1968, 120:1-37.

48. Salt G: Resistance of insect parasitoids to defence reactions of their hosts. Biol Rev 1968, 43:200-232.

49. Van den Bosch R: Encapulation of the eggs of Bathyplectes curculionis (Thomson) in lavae of Hypera brunneipennis (Boheman) and Hypera postica (Gyllenhal). J Insect Pathol 1964, 6:343-367.

50. Blumberg D, Luck RF: Differences in the rates of superparasitism between two strains of Comperiella bifaciata (Howard) parasitizing California red scale - an adaptation to circumvent encapsulation. Ann Entomol Soc Am 1990, 83:591-597.

51. Van Alphen JJM, Visser ME: Superparasitism as an adaptive strategy for insect parasitoids. Ann Rev Entomol 1990, 35:59-79.

52. Vinson SB: How parasitoids deal with the immune-system of their host -an overview. Arch Insect Biochem Physiol 1990, 13:3-27.

53. Ives AR, Schooler SS, Jagar VJ, Knuteson SE, Grbic M, Settle WH: Variability and parasitoid foraging efficiency: a case study of pea aphids and Aphidius ervi. Am Nat 1999, 154:652-673.

54. Grbic M, Strand MR: Shifts in the life history of parasitic wasps correlate with pronounced alterations in early development. Proc Natl Acad Sci USA 1998, 95:1097-1101.

55. Thiboldeaux RL, Hutchison WD, Hogg DB: Species composition of pea aphid (Homoptera, Aphididae) primary and secondary parasitoids in Wisconsin. Can Entomol 1987, 119:1055-1057.

56. Battaglia D, Pennacchio F, Marincola G, Tranfaglia A: Cornicle secretion of Acyrthosiphon pisum (Homoptera, Aphididae) as a contact kairomone for the parasitoid Aphidius ervi (Hymenoptera, Braconidae). Eur J Entomol 1993, 90:423-428.

57. Kunert G, Otto S, Rose USR, Gershenzon J, Weisser WW: Alarm pheromone mediates production of winged dispersal morphs in aphids. Ecol Lett 2005, 8:596-603.

58. Dill LM, Fraser AHG, Roitberg BD: The economics of escape behavior in the pea aphid, Acyrthosiphum pisum. Oecologia 1990, 83:473-478.

59. Dion E, Polin SE, Simon JC, Outreman Y: Symbiont infection affects aphid defensive behaviours. Biol Lett 2011, 7:743-746.

60. Braendle C, Weisser WW: Variation in escape behavior of red and green clones of the pea aphid. J Insect Behav 2001, 14:497-509.

61. McBrien H, Mackauer M: Heterospecific larval competition and host discrimination in 2 species of aphid parasitoids Aphidius ervi and Aphidius smithi. Entomol Exp App/ 1990, 56:145-153.

62. Larocca A, Fanti $P$, Romano VA, Marsicovetere $E$, Isidoro N, Romani $R$, Ruschioni S, Pennacchio F, Battaglia D: Functional bases of hostacceptance behaviour in the aphid parasitoid Aphidius ervi. Physiol Entomol 2007, 32:305-312.

63. Bai B: Conspecific superparasitism in 2 parasitoid wasps, Aphidius ervi Haliday and Aphelinus asychis Walker -reproductive strategies influence host discrimination. Can Entomol 1991, 123:1229-1237.

64. Micha SG, Wellings PW, Morton R: Time-related rejection of parasitized hosts in the aphid parasitoid, Aphidius ervi. Entomol Exp Appl 1992, 62:155-161.

65. Burke G, Fiehn O, Moran N: Effects of facultative symbionts and heat stress on the metabolome of pea aphids. ISME J 2010, 4:242-252.

66. Blackman RL, Eastop VF: In Aphids on the World's Herbaceous Plants and Shrubs. Volume 2. Chichester: John Wiley \& Sons; 2007.

67. Moran NA, Jarvik T: Lateral transfer of genes from fungi underlies carotenoid production in aphids. Science 2010, 328:624-627.

68. Abbot P: Individual and population variation in invertebrates revealed by inter-simple sequence repeats (ISSRs). J Insect Sci 2001, 1:8.

69. Koga R, Tsuchida T, Fukatsu T: Changing partners in an obligate symbiosis: a facultative endosymbiont can compensate for loss of the essential endosymbiont Buchnera in an aphid. Proc $R$ Soc Lond Ser B-BiO Sci 2003, 270.2543-2550.

doi:10.1186/1741-7007-10-11

Cite this article as: Oliver et al:: Parasitic wasp responses to symbiontbased defense in aphids. BMC Biology 2012 10:11. 